2021, Instituto Mexicano de Tecnología del Agua

Tecnología y

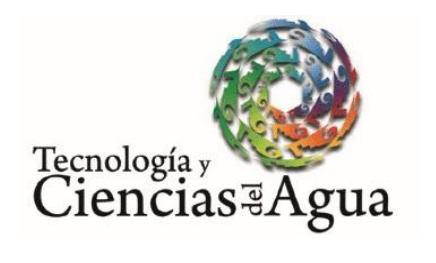

Open Access bajo la licencia CC BY-NC-SA 4.0

(https://creativecommons.org/licenses/by-nc$\mathrm{sa} / 4.0 /$ )

DOI : $10.24850 /$ j-tyca-2021-05-08

Artículos

\title{
Influencia del ángulo y perímetro de impacto de las aspas en un aireador de eje horizontal sobre la transferencia de oxígeno disuelto
}

\section{Influence of the angle and perimeter of contact of the blades in a horizontal axis aerator on the transfer of dissolved oxygen}

César René Blanco-Zúñiga1, ORCID: https://orcid.org/0000-0002-91814944

Nicolas Rojas-Arias², ORCID: https://orcid.org/0000-0003-3358-2484

${ }^{1}$ Universidad Pedagógica y Tecnológica de Colombia, Tunja-Boyacá, Colombia, cesar.blanco@uptc.edu.co

2Universidad Pedagógica y Tecnológica de Colombia, Tunja-Boyacá, Colombia/Graduate Program in Materials Science and Engineering, Department of Materials Engineering, Federal University of São Carlos, São Carlos, Brazil, nicolas.rojas@estudante.ufscar.br

Autor para correspondencia: Nicolas Rojas-Arias, nicolas.rojas@estudante.ufscar.br 
Tecnología y

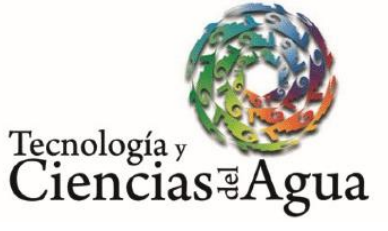

2021, Instituto Mexicano de Tecnología del Agua

Open Access bajo la licencia CC BY-NC-SA 4.0

(https://creativecommons.org/licenses/by-nc$\mathrm{sa} / 4.0 /)$

\section{Resumen}

El oxígeno disuelto (OD) es un parámetro fundamental en los cuerpos de agua que puede suministrarse por medio de agitadores mecánicos de eje horizontal. Sin embargo, la geometría de las aspas y las condiciones operativas pueden influir sobre los costes del proceso. En este trabajo se estudia el efecto del perímetro y ángulo de impacto de las aspas sobre el agua en un prototipo de aireación mecánica de eje horizontal, partiendo del análisis de transferencia de OD. Las mediciones se realizaron en muestras de agua anóxica utilizando dos tipos de aspas con una misma área, variando su perímetro y ángulo de impacto. Otros parámetros que influyen en el proceso de transferencia de OD se controlaron en este estudio. Los resultados muestran que el incremento del perímetro y ángulo de impacto de las aspas con el cuerpo de agua genera una reducción en la eficiencia de aireación estándar (SAE) en el proceso de hasta un $30 \%$ en este estudio, obteniendo los mejores resultados para el prototipo de aspas Tipo II con un valor $\mathrm{K}_{\mathrm{La}}$ de $3.69 \mathrm{~h}^{-1}$ y una SAE de $0.47 \mathrm{kgO}_{2} \cdot \mathrm{kW}^{-1} \mathrm{~h}^{-1}$ al usar un ángulo de impacto de $13.5^{\circ}$. De manera general, la geometría de las aspas es un parámetro fundamental de diseño, el cual influye en la transferencia de oxígeno disuelto en el agua. Los resultados de este estudio pueden contribuir en mejorar la eficiencia en estos sistemas mecánicos, disminuyendo el consumo de energía asociado con los procesos de oxigenación. 
Tecnología y

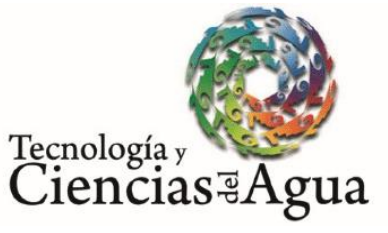

2021, Instituto Mexicano de Tecnología del Agua

Open Access bajo la licencia CC BY-NC-SA 4.0

(https://creativecommons.org/licenses/by-nc$\mathrm{sa} / 4.0 /)$

Palabras clave: aireador mecánico de eje horizontal, oxígeno disuelto (OD), coeficiente de transferencia de oxígeno, eficiencia de aireación estándar(SAE), geometría de las aspas.

\begin{abstract}
Dissolved oxygen (DO) is a fundamental parameter in water bodies that can be supplied using horizontal axis mechanical agitators. However, blade geometry and operating conditions can influence the process cost. In this work, it is studied the effect of the perimeter and angle of impact of the blades on the water in a prototype of horizontal axis mechanical aeration, starting from the DO transfer analysis. Measurements were made on anoxic water samples using two types of blades with the same area, varying their perimeter and angle of impact. Other parameters that influence the DO transfer process were controlled in this work. The results show the increase in the perimeter, and blade impact angle with the water body, which generates a reduction in the standard aeration efficiency (SAE) up to $30 \%$ in the process. In that way, it is obtained the best results for the prototype Type II blades with a $\mathrm{K}_{\mathrm{L} a}$ value of $3.69 \mathrm{~h}^{-1}$, and a SAE of $0.47 \mathrm{kgO}_{2} \cdot \mathrm{kW}^{-1} \mathrm{~h}^{-1}$ when using an impact angle of $13.5^{\circ}$. In general, the geometry of the blades is a fundamental design parameter, which influences the transfer of dissolved oxygen in the water. The results of this study can contribute to improving the efficiency of these mechanical systems, reducing the energy consumption associated with oxygenation processes.
\end{abstract}


Teçnología y

Ciencias $₫$ Agua
2021, Instituto Mexicano de Tecnología del Agua

Open Access bajo la licencia CC BY-NC-SA 4.0

(https://creativecommons.org/licenses/by-nc$\mathrm{sa} / 4.0 /)$

Keywords: Horizontal shaft mechanical aerator, dissolved oxygen (DO), oxygen transfer coefficient, standard aeration efficiency (SAE), blade geometry.

Recibido: $19 / 08 / 2020$

Aceptado: $12 / 10 / 2020$

\section{Introducción}

Los aireadores mecánicos de eje horizontal son sistemas ampliamente utilizados para generar circulación y transferencia de oxígeno disuelto (OD) en cuerpos de agua. Su principal uso se enfoca en lagunas aireadas, zanjas de oxidación y canales de aireación (Tchobanoglous, Louis-Burton, \& Stensel, 2004), aunque también ha sido muy usado en procesos de acuicultura (United States Department of Agriculture \& Service, 2011) y tratamientos de aguas residuales (Barreto et al., 2018; Rojas-Romero, 2010).

Los aireadores mecánicos poseen un cilindro con cerdas, ángulos o láminas de acero o plástico, que promueven la circulación de aire 
Tecnología y

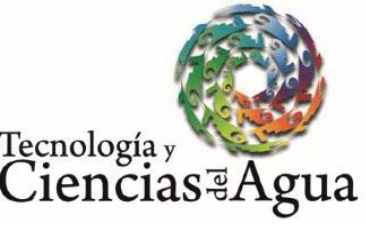

2021, Instituto Mexicano de Tecnología del Agua

Open Access bajo la licencia CC BY-NC-SA 4.0

(https://creativecommons.org/licenses/by-nc$\mathrm{sa} / 4.0 /)$

introduciéndolo dentro de un cuerpo de agua; presenta una tasa de transferencia que varía entre 1.5 y $2.1 \mathrm{kgO}_{2} \cdot \mathrm{kW}^{-1} \mathrm{~h}^{-1}$ en condiciones estándar, y de 0.5 a $1.1 \mathrm{kgO}_{2} \cdot \mathrm{kW}^{-1} \mathrm{~h}^{-1}$ en condiciones de campo (Tchobanoglous et al., 2004). Durante este proceso, el dispositivo es capaz de proporcionar la cantidad suficiente de oxígeno en el medio para mantener las condiciones necesarias para generar la aireación (Barreto et al., 2018; Tahri, Bahafid, Sayel, \& El-Ghachtouli, 2013).

EI OD es probablemente el elemento más crítico de la calidad del agua en diferentes procesos debido a la necesidad de su existencia para la supervivencia de organismos acuáticos (United States Department of Agriculture \& Service, 2011). Sin embargo, el consumo energético que conllevan las etapas de aireación durante el tratamiento de aguas implica un costo entre 50 y $90 \%$ del requerimiento total de energía del proceso (Daw, Hallett, DeWolfe, \& Venner, 2012). Asimismo, la transferencia de OD se ve afectada por diversos factores ambientales (Itano et al., 2019; Ren, Chai, Xue, Anderson, \& Chavez, 2018); físicos (Bao, Peng, Zhou, Zhu, \& Ye, 2018), y microbianos (Torres, Quintero, \& Atehortúa, 2018), entre otros, dificultando la transferencia del oxígeno de atmósfera hacia los cuerpos de agua (Arora \& Keshari, 2018; Bao et al., 2018).

Las características del sistema de aireación como la geometría, tipo y disposición de las aspas también tienen una gran influencia sobre la transferencia de OD (Bahri, Setiawan, Hermawan, \& Junior, 2015), por ejemplo, los tamaños de aspas más grandes, aunque tienden a generar una mayor aireación, requieren mayor consumo de potencia debido a una mayor fuerza de arrastre, lo que aumenta el costo operativo del proceso, 
Tecnología y

Ciencias $\approx$ Agua
2021, Instituto Mexicano de Tecnología del Agua

Open Access bajo la licencia CC BY-NC-SA 4.0

(https://creativecommons.org/licenses/by-nc$\mathrm{sa} / 4.0 /)$

incluyendo electricidad y consumo de combustible (Qiu et al., 2018; Samsul-Bahri \& Wawan-Hermawan, 2015).

El objetivo de este trabajo se enfoca en estudiar la influencia del perímetro de impacto, manteniendo un mismo valor de área, de dos diferentes tipos de aspas, controlando la velocidad de rotación (RPM), así como el ángulo de impacto sobre el cuerpo de agua. Los ensayos son realizados a una escala de laboratorio, centrándose principalmente en el efecto que puede generar la variación del perímetro del aspa sobre la transferencia de OD.

\section{Materiales y métodos}

Los ensayos se llevaron a cabo en condiciones controladas usando muestras de 44 I de agua potable, manteniendo un área de contacto con la atmósfera de $0.1924 \mathrm{~m}^{2}$ y una profundidad de $0.24 \mathrm{~m}$. La concentración de saturación de oxígeno en las muestras de agua fue de $7.5 \mathrm{mg} \cdot \mathrm{l}^{-1}\left(\mathrm{C}_{\mathrm{s}}\right)$, obtenida mediante oxímetro Hach-flexi HQ30d-US a una temperatura ambiente de $17^{\circ} \mathrm{C}$, presión atmosférica de $740 \mathrm{hPa}$ y una altura de 2800 msnm. Este valor es comparable con la temperatura y presión atmosférica en la zona de medición (Sander, 2015). Otros parámetros propios del 
Tecnología y

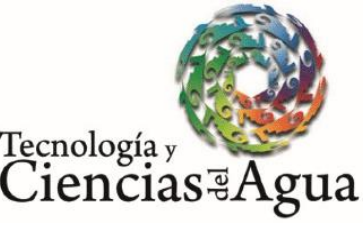

2021, Instituto Mexicano de Tecnología del Agua

Open Access bajo la licencia CC BY-NC-SA 4.0

(https://creativecommons.org/licenses/by-nc$\mathrm{sa} / 4.0 /)$

agua, como conductividad, salinidad, temperatura y $\mathrm{pH}$ se controlaron en un proceso experimental. La remoción del OD se hizo mediante la aplicación de $3.35 \mathrm{~g}$ de sulfito de sodio $\left(\mathrm{Na}_{2} \mathrm{SO}_{3}\right)$ y $0.05 \mathrm{mg} \cdot \mathrm{l}^{-1}$ de cloruro de cobalto anhidro $\left(\mathrm{CoCl}_{2}\right)$ como agente catalizador (Engineers, A. S. of C., 2007; Uby, 2019). Los pesos se midieron en una balanza Ohaus SPX123 con precisión de $0.001 \mathrm{~g}$. El fluido se agitó a 10 RPM hasta asegurar la remoción total de oxígeno.

El montaje de aireación se presenta en la Figura 1. Fue utilizado un soporte de aspas intercambiable construido por impresión 3D, utilizando PETG (tereftalato de polietileno glicol). El sistema se puso en marcha usando un motorreductor Greartisan DC de $12 \mathrm{~V}$ con una velocidad de rotación máxima de 50 RPM. Las aspas utilizadas en el ensayo fueron de lámina de zinc galvanizado con espesorde $0.6 \mathrm{~mm}$. El primer tipo de aspa se compone de tres secciones de $18 \times 2.2 \mathrm{~cm}$ cada una (Tipo I) y un perímetro de impacto de $114.6 \mathrm{~cm}$. El segundo tipo de aspa es de una sola pieza con dimensión de $18 \times 6.6 \mathrm{~cm}$ y un perímetro de impacto con el agua de $42.6 \mathrm{~cm}$ (Tipo II). La configuración del sistema se realizó para impactar las aspas con la superficie del agua a $180^{\circ}$ (paralelo con la superficie del agua) y $13.5^{\circ}$, variando el sentido de rotación. La potencia máxima necesaria parar la operación del sistema se calculó a partir de una velocidad de rotación de $20 \pm 1$ RPM, buscando evitar pérdidas del fluido por efectos de derrames o salpicaduras, poco eficaces en los procesos de transferencia de OD (Bahri, Jufriadi, Hamdani, \& Anwar, 2019). 
Tecnología y

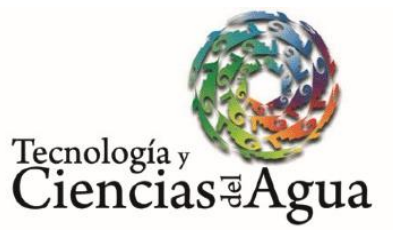

2021, Instituto Mexicano de Tecnología del Agua

Open Access bajo la licencia CC BY-NC-SA 4.0

(https://creativecommons.org/licenses/by-nc$\mathrm{sa} / 4.0 /)$
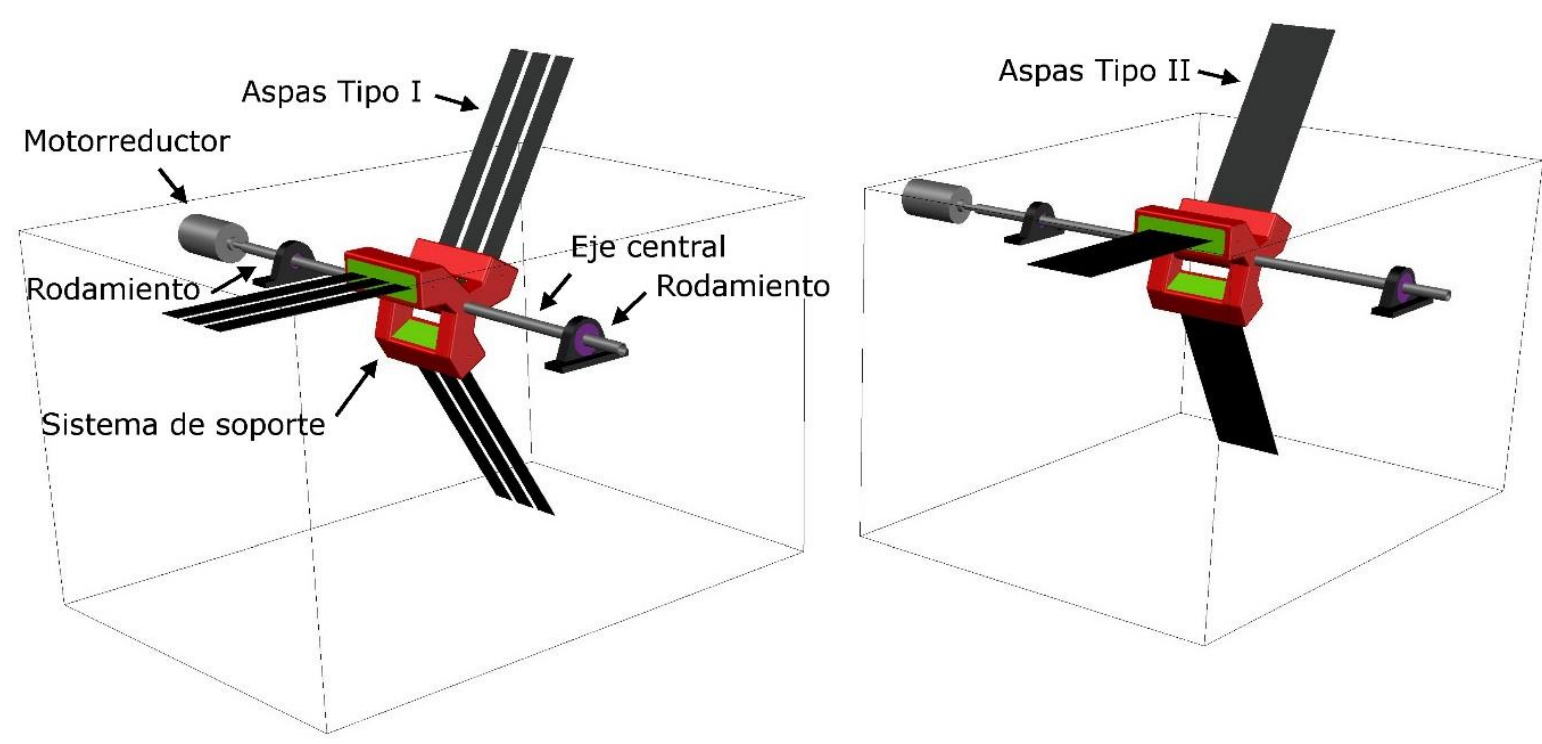

Figura 1. Esquema de los aireadores mecánicos de eje horizontal utilizados en este estudio usando el sistema de aspas Tipo I (izquierda) y el sistema de aspas Tipo II (derecha).

La concentración de OD en las muestras de agua se midió con oxímetro en intervalos de cinco minutos durante una hora y después en intervalos de 15 minutos hasta alcanzar una concentración de saturación del 98 \% de la concentración inicial (Engineers, A. S. of C., 2007). Las mediciones se realizaron en las diagonales opuestas del montaje para cada tiempo de medida a una profundidad de $5 \mathrm{~cm}$. Se hicieron seis réplicas para cada ensayo, con el fin de asegurar la validez y replicabilidad del proceso.

Los datos obtenidos en este estudio se analizaron mediante modelos de regresión no lineal; se evaluó el rendimiento del proceso de aireación. 
Tecnología y

Ciencias $₫$ Agua
2021, Instituto Mexicano de Tecnología del Agua

Open Access bajo la licencia CC BY-NC-SA 4.0

(https://creativecommons.org/licenses/by-nc$\mathrm{sa} / 4.0 /)$

La tasa de transferencia de OD (SOTR), el coeficiente de transferencia $\left(\mathrm{K}_{\mathrm{L}} \mathrm{a}\right)$, y la eficiencia estándar de aireación ( $\mathrm{SAE}$, por sus siglas en inglés) se llevaron a cabo mediante los cálculos presentados por Rojas-Romero (2010), y Roy, Moulick y Mal (2017), utilizando un factor de corrección de temperatura para el valor de $\mathrm{K}_{\mathrm{L}} \mathrm{a}$. La transferencia se puede expresar de la siguiente manera:

$\frac{\mathrm{dC}}{\mathrm{dt}}=\mathrm{K}_{\mathrm{L}} \mathrm{a}\left(\mathrm{C}_{\infty}^{*}-\mathrm{C}\right)$

Donde:

$\mathrm{K}_{\mathrm{L}} \mathrm{a}=$ coeficiente de transferencia de masa volumétrico $(1 / \mathrm{T})$.

$\mathrm{C}_{\infty}^{*}=$ concentración final promedio de OD alcanzada en un tiempo infinito $\left(\mathrm{mg} \cdot \mathrm{l}^{-1}\right)$.

$\mathrm{C}=$ concentración promedio efectiva de OD en la fase líquida.

La forma integrada se convierte en:

$\ln \frac{\mathrm{C}_{\infty}^{*}-\mathrm{C}_{\mathrm{o}}}{\mathrm{C}_{\infty}^{*}-\mathrm{C}}=\mathrm{K}_{\mathrm{L}} \mathrm{a}\left(\mathrm{t}-\mathrm{t}_{0}\right)$

$C=C_{\infty}^{*}-\left(C_{\infty}^{*}-C_{0}\right) \cdot \exp \left[K_{L} a\left(t-t_{0}\right)\right]$ 
Tecnología y

Ciencias Agua
2021, Instituto Mexicano de Tecnología del Agua

Open Access bajo la licencia CC BY-NC-SA 4.0

(https://creativecommons.org/licenses/by-nc$\mathrm{sa} / 4.0 /)$

Donde:

$\mathrm{C}_{0}=$ concentración inicial de OD, cuando $\mathrm{t}=\mathrm{t}_{0}$.

Las ecuaciones (2) y (3) se utilizan para pruebas en agua limpia, en estado no estacionario, para determinar la capacidad de transferencia de equipos de aireación y pueden ser aplicadas a diferentes sistemas de aireación (Liu, Shi, Wang, Fan, \& Shi, 2013). Debido a que la temperatura varía durante el proceso de experimentación, el coeficiente de transferencia de masa volumétrico KLa debe ser convertido a temperatura estándar $\left(T=20^{\circ} \mathrm{C}\right)$ por medio de la Ecuación (4):

$\mathrm{K}_{\mathrm{L}} \mathrm{a}_{20}=1.024^{(20-\mathrm{t})}$

La tasa de transferencia estándar de oxígeno $\left(\mathrm{SOTR}_{20} \mathrm{kgO}_{2} \cdot \mathrm{h}^{-1}\right)$ señala la cantidad de oxígeno transferido por hora en condiciones estándar, la cual puede calcularse con base en la siguiente ecuación:

SOTR $=\mathrm{K}_{\mathrm{L}} \mathrm{a}_{20} \cdot \mathrm{C}_{\mathrm{s}, 20} \cdot \mathrm{V}$

Donde: 
2021, Instituto Mexicano de Tecnología del Agua

Open Access bajo la licencia CC BY-NC-SA 4.0

(https://creativecommons.org/licenses/by-nc$\mathrm{sa} / 4.0 /)$

$\mathrm{K}_{\mathrm{L}} \mathrm{a}_{20}=$ coeficiente de transferencia de masa volumétrico $(1 / \mathrm{T})$, a una temperatura de $20^{\circ} \mathrm{C}$.

$\mathrm{C}_{\mathrm{s}, 20}=$ concentración de saturación para un estado estable a una temperatura de $20^{\circ} \mathrm{C}$.

$\mathrm{V}=$ volumen del líquido.

La eficiencia estándar de aireación (SAE) se expresa como la transferencia de oxígeno por unidad de potencia, tal y como se señala en la siguiente ecuación:

$\mathrm{SAE}=\frac{\text { SOTR }}{\text { Potencia de entrada }}$

Donde:

SOTR $=$ tasa de transferencia estándar de oxígeno $\left(\mathrm{kgO}_{2} \cdot \mathrm{h}^{-1}\right)$.

Potencia de entrada $=$ potencia consumida en kilovatios $(\mathrm{kW})$.

La potencia en vatios [kW] del motorreductor se calculó como el producto de la tensión eléctrica (voltaje) multiplicada por la intensidad de la corriente eléctrica (amperaje) con base en la siguiente ecuación: 
2021, Instituto Mexicano de Tecnología del Agua

Donde:

$\mathrm{P}=$ potencia expresada en vatios.

$\mathrm{V}=$ voltaje

$\mathrm{A}=$ amperaje.

\section{Resultados y discusión}

Se hicieron mediciones de transferencia de OD en cuerpos de agua anóxica utilizando dos tipos de aspa que contenían una misma área superficial, pero un perímetro de contacto diferente, con un ángulo de impacto de $180^{\circ}$ y $13.5^{\circ}$. Los resultados obtenidos permitieron el análisis de los datos a partir de gráficos y relaciones matemáticas que determinan la eficiencia del proceso de transferencia realizado para cada tipo de muestra. 
Tecnología y

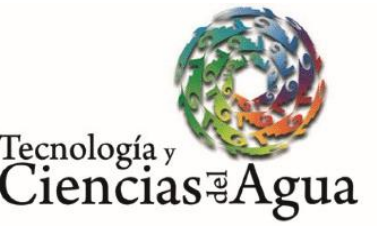

2021, Instituto Mexicano de Tecnología del Agua

Open Access bajo la licencia CC BY-NC-SA 4.0

(https://creativecommons.org/licenses/by-nc$\mathrm{sa} / 4.0 /)$

Se observa una alta tasa de transferencia de OD durante los primeros minutos en las muestras de agua, reduciéndose gradualmente hasta llegar a un valor máximo de saturación generando una fase de estabilización. El manejo de muestras de agua en condiciones de anoxia e hipoxia experimentará una mayor velocidad de saturación con oxígeno en su etapa inicial debido a la diferencia existente entre la presión parcial de $\mathrm{OD}$ en el agua y el $\mathrm{O}_{2}$ atmosférico (Abdelrahman \& Boyd, 2018; Sander, 2015). Al llegar al punto de concentración máxima de saturación del OD en el agua, la velocidad transferencia del oxígeno se verá reducida, generando un comportamiento tipo Arrhenius hasta llegar a un punto de equilibrio, penalizando el consumo de energía del sistema aireador (Bahadori \& Vuthaluru, 2010).

La Figura 2 muestra la variación de la concentración del OD del agua en función del tiempo de agitación. Las aspas giran e impactan el agua con un ángulo de entrada de $13.5^{\circ}$ y $180^{\circ}$. Se obtuvieron valores cercanos al $95 \%$ de la concentración inicial de OD durante los primeros 60 minutos, para luego generar una atenuación gradual en la tasa de transferencia de OD hasta lograr $98 \%\left(7.35 \mathrm{mg} \cdot \mathrm{I}^{-1}\right)$ de la concentración inicial a los 120 minutos de iniciar el experimento. 
Teçnología y

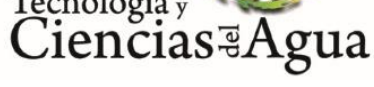

2021, Instituto Mexicano de Tecnología del Agua Open Access bajo la licencia CC BY-NC-SA 4.0 (https://creativecommons.org/licenses/by-nc$\mathrm{sa} / 4.0 /)$

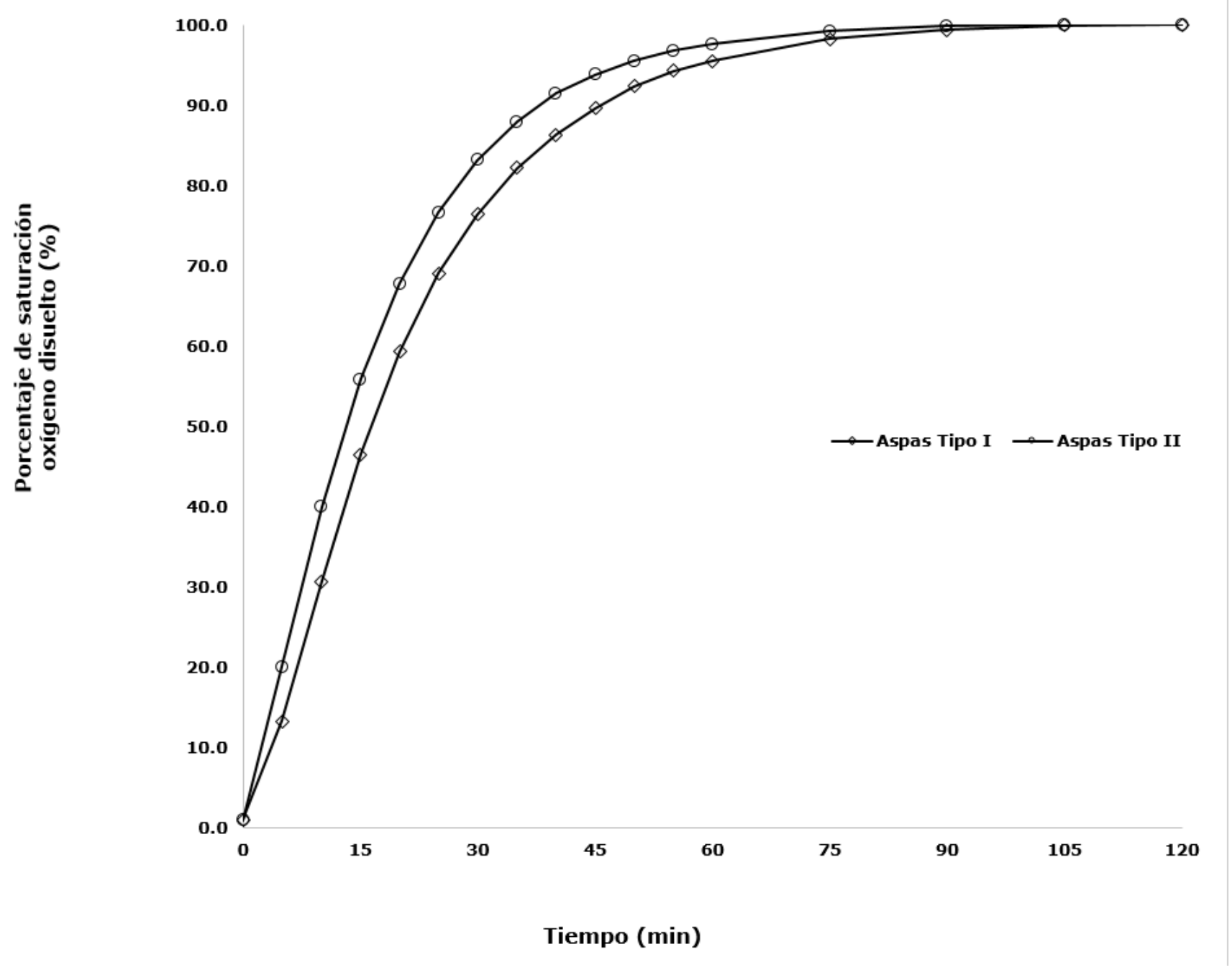

Figura 2. Curvas de porcentaje de saturación del OD en las muestras de agua trabajadas con un ángulo de impacto de $13.5^{\circ}$ y $180^{\circ}$.

Al usar un ángulo de $180^{\circ}$, las aspas Tipo II generan un mayor desplazamiento de agua al sumergirse. Esta situación favorece una mayor agitación, atrapamiento y transporte de aire dentro del cuerpo de agua, 
Tecnología y

Ciencias $₫$ Agua
2021, Instituto Mexicano de Tecnología del Agua

Open Access bajo la licencia CC BY-NC-SA 4.0

(https://creativecommons.org/licenses/by-nc$\mathrm{sa} / 4.0 /)$

cómo se observa en la Figura 2. Por otra parte, la aplicación de aspas con un mayor perímetro de contacto reduce la posibilidad de atrapar y transportar una mayor cantidad de aire hacia el interior del cuerpo de agua, reduciendo la tasa de transferencia de OD por unidad de tiempo.

La reducción del ángulo en el que las aspas impactan el agua permite un aumento en la tasa de transferencia de OD en el fluido. La relación de transferencia de OD en función del tiempo puede observarse en la Figura 3. La fuerza ejercida por unidad de área se reduce al impactar parcialmente las aspas con el cuerpo de agua, requiriendo un menor suministro de energía para mantener el sistema trabajando a una velocidad de rotación constante. Una inclinación de las aspas puede contribuir a un mayor atrapamiento de aire dentro del sistema de forma comparable a lo presentado por Tian, Xu, Chen, Liu y Zhang (2018). Esta reducción en la fuerza ejercida se ve reflejada en la energía requerida por el sistema para mantener una velocidad de rotación estable (Bahri et al., 2015; Bahri et al., 2019). 
Tecnología y

Ciencias ฐึgua
2021, Instituto Mexicano de Tecnología del Agua

Open Access bajo la licencia CC BY-NC-SA 4.0

(https://creativecommons.org/licenses/by-nc$\mathrm{sa} / 4.0 /)$

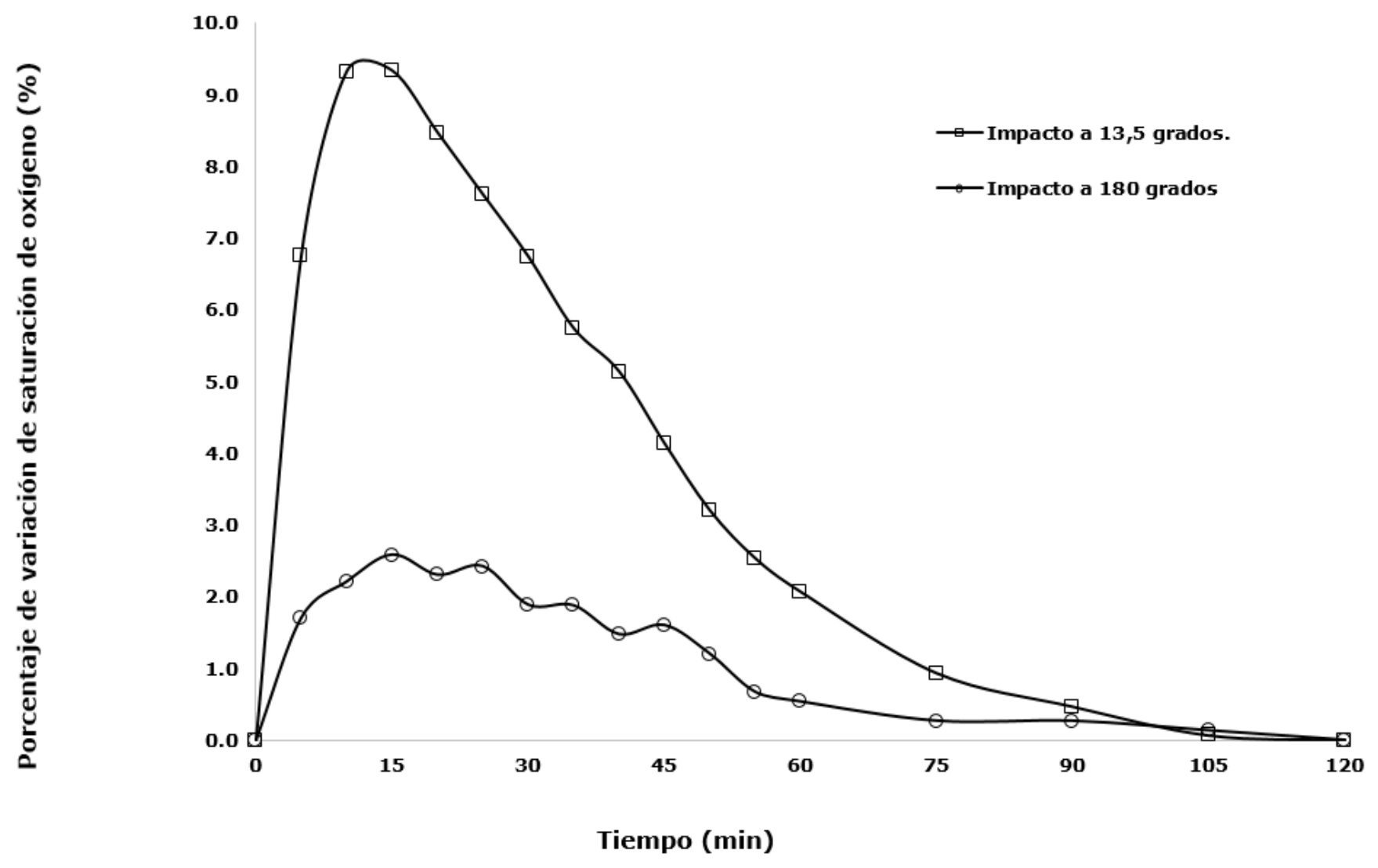

Figura 3. Variación en el porcentaje de transferencia de OD por tiempo de medición entre los dos sistemas trabajados con ángulo de impacto del aspa de $13.5^{\circ}$ y $180^{\circ}$, respectivamente.

Los coeficientes de transferencia de masa volumétrica $\mathrm{KLa}\left(\mathrm{h}^{-1}\right)$ se calcularon utilizando el método de regresión no lineal (Engineers, A. S. of C., 2007). Estos datos permitieron determinar la eficiencia del proceso de transferencia en función del consumo energético. El resumen de los valores obtenidos en este estudio se presenta en la Tabla 1. Se observa 
Tecnología y

Ciencias $\stackrel{\Xi}{\Im}$ gua
2021, Instituto Mexicano de Tecnología del Agua

Open Access bajo la licencia CC BY-NC-SA 4.0

(https://creativecommons.org/licenses/by-nc$\mathrm{sa} / 4.0 /)$

un coeficiente KLa para el sistema de aspas Tipo I con un valor equivalente de $3.00 \mathrm{~h}^{-1}$ y $2.87 \mathrm{~h}^{-1}$ con un ángulo de impacto de $13.5^{\circ}$ y $180^{\circ}$, respectivamente. Los valores de KLa obtenidos usando las aspas de Tipo II muestran un valor de $3.69 \mathrm{~h}^{-1}$ (23\% mayor) y $3.06 \mathrm{~h}^{-1}$ (6.6 \% mayor) con relación al primer dispositivo. Independientemente de la variación de potencia suministrada, la geometría de las aspas es un parámetro de diseño muy importante que puede influir de manera trascendental en la agitación del agua y en transferencia de oxígeno disuelto.

Tabla 1. Resumen de los datos obtenidos en este estudio para los sistemas de aireación con aspas Tipo I y Tipo II.

\begin{tabular}{|l|c|c|c|c|}
\hline \multirow{2}{*}{ Ángulo de impacto } & \multicolumn{2}{c|}{ Aspa Tipo I } & \multicolumn{2}{c|}{ Aspa Tipo II } \\
\cline { 2 - 5 } & $\mathbf{1 3 . 5 ^ { \circ }}$ & $\mathbf{1 8 0}^{\circ}$ & $\mathbf{1 3 . 5}^{\circ}$ & $\mathbf{1 8 0}^{\circ}$ \\
\hline Voltaje $(\mathrm{V})$ & 6 & 6 & 6 & 6 \\
\hline Amperaje $(\AA)$ & 0.46 & 0.53 & 0.53 & 0.63 \\
\hline Potencia $(\mathrm{W})$ & 2.76 & 3.18 & 3.18 & 3.78 \\
\hline KLa $\left(\mathrm{h}^{-1}\right)$ & 3.00 & 2.87 & 3.69 & 3.06 \\
\hline Velocidad de rotación (RPM) & 21 & 20 & 21 & 20 \\
\hline dc/dt $\left(\mathrm{g} \cdot \mathrm{h}^{-1} \mathrm{~m}^{-3}\right)$ & 27.62 & 26.41 & 33.98 & 28.19 \\
\hline SOTR $\left(\mathrm{kgO}_{2} \cdot \mathrm{h}^{-1}\right)$ & 1.22 & 1.16 & 1.49 & 1.24 \\
\hline SAE $\left(\mathrm{kgO}_{2} \cdot \mathrm{kW}^{-1} \mathrm{~h}^{-1}\right)$ & 0.44 & 0.37 & 0.47 & 0.33 \\
\hline
\end{tabular}


Tecnología y

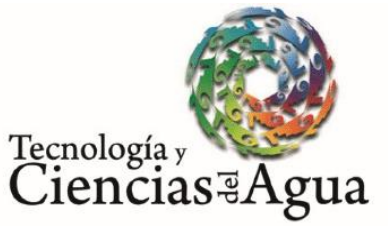

2021, Instituto Mexicano de Tecnología del Agua

Open Access bajo la licencia CC BY-NC-SA 4.0

(https://creativecommons.org/licenses/by-nc$\mathrm{sa} / 4.0 /)$

Los valores de SAE también se obtuvieron en este estudio al observar el efecto en la variación del perímetro y ángulo de impacto de las aspas sobre consumo energético que conlleva transferir OD a los cuerpos de agua. El área superficial de las aspas para las dos situaciones experimentales fue la misma; sin embargo, las aspas Tipo II requirieron un mayor consumo de energía al momento de impactar y romper la superficie del agua. Este consumo de energía se ve reducido al utilizar un ángulo de impacto de $13.5^{\circ}$, logrando valores de potencia similares a los alcanzados en el sistema con aspas Tipo I y ángulo de impacto a $180^{\circ}$.

La reducción en la fuerza y torque ejercido para mantener una velocidad de rotación constante se redujo de modo considerable, mejorando la SAE en un 19 y $42 \%$ para los sistemas con aspas Tipo I y Tipo II, respectivamente, al reducir el ángulo de impacto. Se presume que los espacios intermedios que separan las aspas Tipo I permiten una menor resistencia al avance del flujo del agua durante el momento del impacto. Esta situación reduce la fuerza necesaria para que estas aspas penetren la superficie del agua, disminuyendo así el consumo de energía asociado con esta situación mientras se mantiene una velocidad de rotación constante del sistema.

La SOTR depende del número de aspas y se incrementa con un aumento de estas, así como de la velocidad de rotación del aireador (Roy et al., 2017). Sin embargo, en este caso podemos observar que el tipo y geometría del aspa pueden afectar este valor. Los valores de SAE muestran que el mejor comportamiento se obtuvo para la configuración de aspas Tipo II con un ángulo de impacto de $13.5^{\circ}$; mientras el peor 
2021, Instituto Mexicano de Tecnología del Agua

Open Access bajo la licencia CC BY-NC-SA 4.0

(https://creativecommons.org/licenses/by-nc$\mathrm{sa} / 4.0 /)$

comportamiento puede observarse en el mismo sistema al variar el ángulo a $180^{\circ}$.

El estudio permitió observar el comportamiento de las aspas al sumergirse en el cuerpo de agua. Se observa un atrapamiento de aire durante la inmersión de las aspas dentro del cuerpo de agua. Las imágenes obtenidas se presentan en la Figura 4. Este comportamiento se mantiene independiente del ángulo de impacto del aspa, teniendo una mayor importancia la forma del aspa trabajada. 
Tecnología y

Ciencias $\stackrel{乛}{\approx}$ gua
2021, Instituto Mexicano de Tecnología del Agua

Open Access bajo la licencia CC BY-NC-SA 4.0

(https://creativecommons.org/licenses/by-ncsa/4.0/)
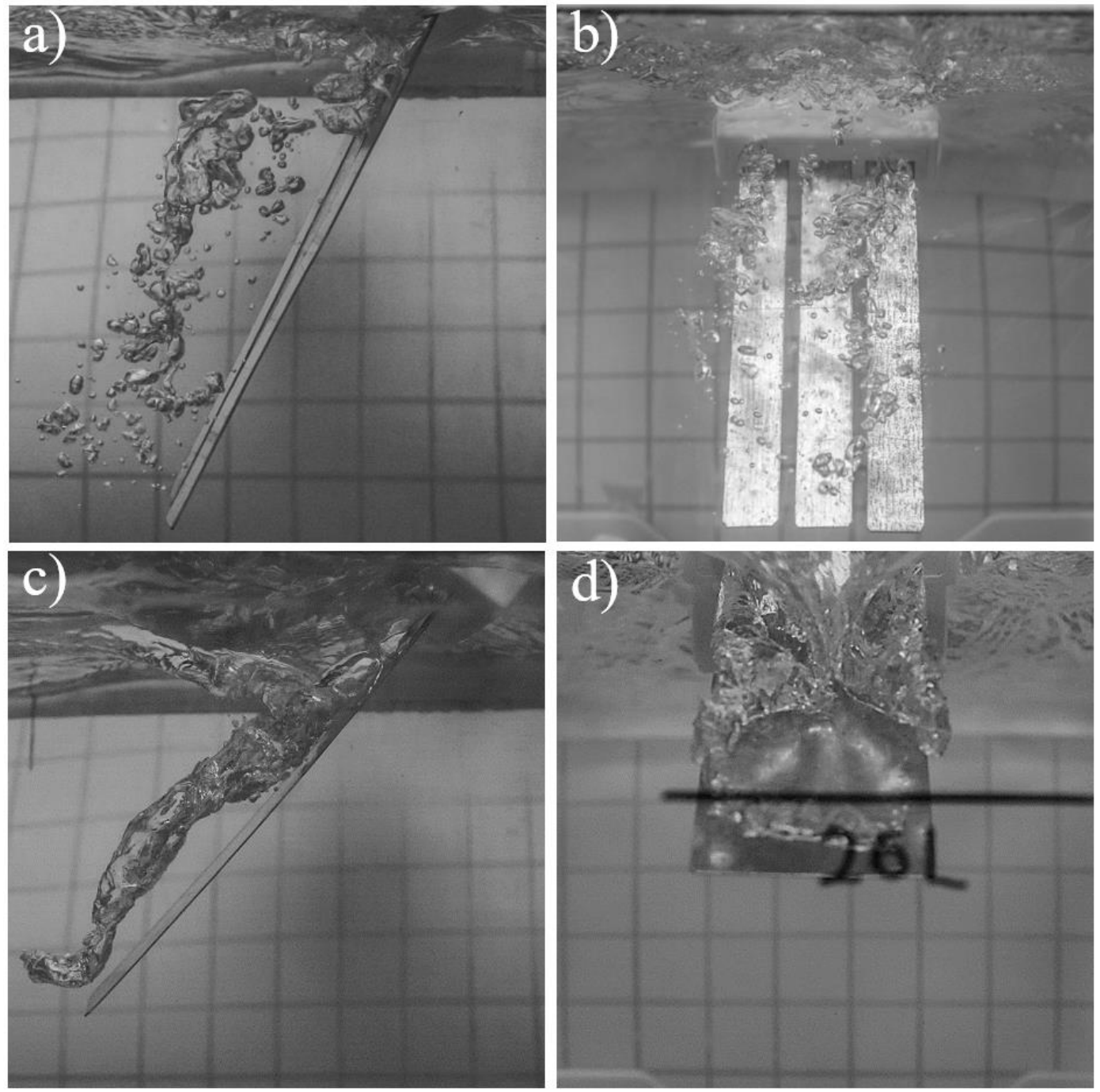

Figura 4. Comportamiento del aire atrapado bajo el agua en los sistemas de aspas trabajados. a-b) sistemas de aspas Tipo I; c-d) sistema de aspas Tipo II.

Dependiendo del tipo de aspa utilizado, este comportamiento presenta una variación en el atrapamiento de aire, por ejemplo, en la 
Tecnología y

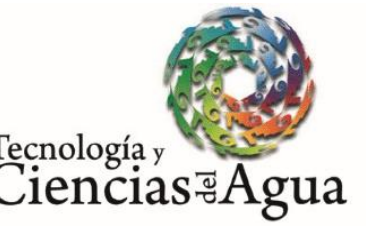

2021, Instituto Mexicano de Tecnología del Agua

Open Access bajo la licencia CC BY-NC-SA 4.0

(https://creativecommons.org/licenses/by-nc$\mathrm{sa} / 4.0 /)$

Figura 4 (c y d) se aprecia un atrapamiento en forma de vórtice al aplicar un sistema con aspas Tipo II. Estas aspas, al poseer un mayor ancho, generan más depresión al momento de impactar y sumergirse en el cuerpo del agua, concentrando el retorno del agua a su estado original a través de los bordes laterales. Por otra parte, el aire retenido toma forma de varios vórtices que se ubican en los extremos de las aspas. Los vórtices son desplazados dentro del agua por el movimiento inercial de rotación que mantiene el sistema. En el caso del sistema de aspas Tipo I (Figura 4 (a y b)), el aire atrapado termina por colisionar, desintegrándose a sí mismo, para conformar cúmulos de burbujas, las cuales terminan por alcanzar la superficie del agua. Un mejor entendimiento de esta situación puede favorecer el diseño de sistemas aireadores mecánicos más eficaces frente al consumo de energía y con una mayor tasa de transferencia de OD.

\section{Conclusiones}

Se ha estudiado el efecto del borde y ángulo de contacto en dos tipos de aspa utilizados en un sistema de aireación mecánico de eje horizontal en cuerpos de agua anóxica. Se observó que la importancia de la geometría 
Tecnología y

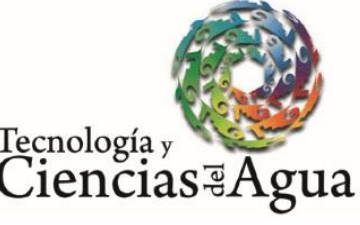

2021, Instituto Mexicano de Tecnología del Agua

Open Access bajo la licencia CC BY-NC-SA 4.0

(https://creativecommons.org/licenses/by-nc$\mathrm{sa} / 4.0 /)$

y variación del perímetro de contacto del aspa, así como el ángulo al momento de impactar con el cuerpo de agua presentan una gran influencia sobre el proceso de transferencia del OD, así como la eficiencia energética, en términos de cantidad de OD transferido por consumo de energía durante el proceso de aireación.

La transferencia de oxígeno atmosférico en OD se generó solo por los fenómenos de agitación de la superficie y el atrapamiento de aire dentro del cuerpo de agua. Se eliminaron los efectos de transferencia dados por levantamiento y la salpicadura del fluido por encima de la superficie del agua al mantener una velocidad de rotación baja. La geometría de las aspas en un aireador mecánico de eje horizontal puede generar un comportamiento predecible del aire atrapado dentro de un cuerpo de agua incluso cuando se presenta agitación o turbulencia sobre la misma superficie del fluido.

El efecto del perímetro y ángulo de impacto de las aspas sobre el cuerpo de agua se ve reflejado en la eficiencia y consumo energético del proceso. La reducción del ángulo de contacto genera una mejora en el proceso en costes energéticos, principalmente en sistemas de aspas Tipo II. Además, se observó la formación de vórtices y atrapamiento de burbujas de aire dentro de los cuerpos de agua, los cuales pueden contribuir en el proceso de transferencia. Se espera estudiar más a profundidad este efecto en investigaciones futuras.

Los autores expresan su motivación en el desarrollo de esta investigación por las afectaciones que el cambio climático puede ejercer sobre los cuerpos de agua, como es la desoxigenación por aumento de la 
Tecnología y

Ciencias $\approx$ Agua
2021, Instituto Mexicano de Tecnología del Agua

Open Access bajo la licencia CC BY-NC-SA 4.0

(https://creativecommons.org/licenses/by-nc$\mathrm{sa} / 4.0 /)$

temperatura. Este problema podría afectar fuertemente ecosistemas acuáticos en diferentes entornos, así como las actividades comerciales que se realizan en estanques y lagunas, como es el caso de los procesos de acuicultura. La investigación y el desarrollo de nuevas tecnologías puede favorecer la mitigación de esta problemática asociada con los cuerpos de agua.

\section{Agradecimientos}

Los autores desean agradecer al Departamento de Ingeniería Ambiental de la Universidad Pedagógica y Tecnológica de Colombia por su participación en este trabajo. Uno de los autores (NRA) desea agradecer especialmente a esta institución por su invitación para contribuir en este trabajo.

\section{Referencias}

Abdelrahman, H. A., \& Boyd, C. E. (2018). Effects of mechanical aeration on evaporation rate and water temperature in aquaculture ponds. Aquaculture Research, 49(6), 2184-2192. Recuperado de https://doi.org/10.1111/are.13674

Arora, S., \& Keshari, A. K. (2018). Estimation of re-aeration coefficient using MLR for modelling water quality of rivers in urban environment. Groundwater for Sustainable Development, 7, 430-435. Recuperado de https://doi.org/10.1016/j.gsd.2017.11.006 
Tecnología y

Ciencias $\approx$ Agua
2021, Instituto Mexicano de Tecnología del Agua

Open Access bajo la licencia CC BY-NC-SA 4.0

(https://creativecommons.org/licenses/by-nc$\mathrm{sa} / 4.0 /)$

Bahadori, A., \& Vuthaluru, H. B. (2010). Simple Arrhenius-type function accurately predicts dissolved oxygen saturation concentrations in aquatic systems. Process Safety and Environmental Protection, 88(5), 335-340.

Recuperado

de https://doi.org/10.1016/j.psep.2010.05.002

Bahri, S., Jufriadi, Hamdani, \& Anwar. (2019). The ineffectiveness of water splash on paddlewheel aerator. IOP Conference Series: Earth and Environmental Science, 268, 012162. Recuperado de https://doi.org/10.1088/1755-1315/268/1/012162

Bahri, S., Setiawan, R. P. A., Hermawan, W., \& Junior, M. Z. (2015). Simulation on blade geometry and operational condition toward torque requirement and drag force in paddle wheel aerator. International Journal of Scientific \& Engineering Research, 6(2), 812816.

Bao, W., Peng, Z., Zhou, D., Zhu, S., \& Ye, Z. (2018). Performance evaluation of an intensive pond aquaculture system for commercial freshwater fish production. Recuperado

de https://doi.org/10.13031/aim.201801093

Barreto, C. M., Ochoa, I. M., Garcia, H. A., Hooijmans, C. M., Livingston, D., Herrera, A., \& Brdjanovic, D. (2018). Sidestream superoxygenation for wastewater treatment: Oxygen transfer in clean water and mixed liquor. Journal of Environmental Management, 219, 125-137. Recuperado de https://doi.org/10.1016/j.jenvman.2018.04.035 
Tecnología y

Ciencias $₫$ Agua
2021, Instituto Mexicano de Tecnología del Agua

Open Access bajo la licencia CC BY-NC-SA 4.0

(https://creativecommons.org/licenses/by-nc$\mathrm{sa} / 4.0 /)$

Daw, J., Hallett, K., DeWolfe, J., \& Venner, I. (2012). Energy efficiency strategies for municipal wastewater treatment facilities (Technical Report NREL/TP-7A30-53341 January 2012). Golden, USA: National Renewable Energy Laboratory. Recuperado de https://www.nrel.gov/docs/fy12osti/53341.pdf

Engineers, A. S. of C. (2007). ASCE Standar: Measurement of Oxygen Transfer in Clean Water(2-06). Reston, USA: Engineers, A. S. of C.

Itano, T., Inagaki, T., Nakamura, C., Hashimoto, R., Negoro, N., Hyodo, J., \& Honda, S. (2019). Water circulation induced by mechanical aerators in a rectangular vessel for shrimp aquaculture. Aquacultural Engineering, 85, 106-113. Recuperado de https://doi.org/10.1016/j.aquaeng.2019.03.006

Liu, Y., Shi, H., Wang, Z., Fan, L., \& Shi, H. (2013). Approach to enhancing nitrogen removal performance with fluctuation of influent in an oxidation ditch system. Chemical Engineering Journal, 219, 520526. Recuperado de https://doi.org/10.1016/j.cej.2012.09.085

Qiu, Y., Zhang, C., Li, B., Li, J., Zhang, X., Liu, Y., Liang, P., \& Huang, X. (2018). Optimal surface aeration control in full-scale oxidation ditches through energy consumption analysis. Water (Switzerland), 10(7). Recuperado de https://doi.org/10.3390/w10070945

Ren, A. S., Chai, F., Xue, H., Anderson, D. M., \& Chavez, F. P. (2018). A sixteen-year decline in dissolved oxygen in the Central California current. Scientific Reports, 8(1), 1-9. Recuperado de https://doi.org/10.1038/s41598-018-25341-8 
Tecnología y

Ciencias $\approx$ Agua
2021, Instituto Mexicano de Tecnología del Agua

Open Access bajo la licencia CC BY-NC-SA 4.0

(https://creativecommons.org/licenses/by-nc$\mathrm{sa} / 4.0 /)$

Rojas-Romero, J. A. (2010). Tratamiento de aguas residuales. Teoría y principios de diseño ( $3^{a}$ ed.). Escuela Colombiana de Ingeniería. Recuperado de papers://73c07e2e-9294-4a94-b11eb804a5393384/Paper/p187

Roy, S. M., Moulick, S., \& Mal, B. C. (2017). Design characteristics of spiral aerator. Journal of the World Aquaculture Society, 48(6), 898908. Recuperado de https://doi.org/10.1111/jwas.12410

Samsul-Bahri, R. P. A. S., \& Wawan-Hermawan, M. Z. J. (2015). Design and simulation of paddle wheel aerator with movable blades. International Journal of Engineering Research and Technology (IJERT), 4(2), 994-999.

Sander, R. (2015). Compilation of Henry's law constants (version 4.0) for water as solvent. Atmospheric Chemistry and Physics, 15(8), 43994981.

Tahri, N., Bahafid, W., Sayel, H., \& El-Ghachtouli, N. (May, 2013). Biodegradation: Involved microorganisms and genetically engineered microorganisms. Biodegradation. Life of Science,. Recuperado de https://doi.org/10.5772/56194

Tchobanoglous, G., Louis-Burton, F., \& Stensel, H. D. (2004). Wastewater engineering treatment and reuse ( $4^{\text {th }}$ ed.). Boston, USA: McGraw Hill.

Tian, L., Xu, Z., Chen, L., Liu, Y., \& Zhang, T. (2018). Study on oxygen gas holdup and kinetics using various types of paddles during marmatite leaching process. Hydrometallurgy, 180, 158-171. Recuperado de https://doi.org/10.1016/j.hydromet.2018.06.011 
Tecnología y

Ciencias $₫$ Agua
2021, Instituto Mexicano de Tecnología del Agua

Open Access bajo la licencia CC BY-NC-SA 4.0

(https://creativecommons.org/licenses/by-nc$\mathrm{sa} / 4.0 /)$

Torres, A., Quintero, J., \& Atehortúa, L. (2018). Determination of the specific oxygen uptake rate in microorganisms including electrode time response. Revista de la Facultad de Ingeniería, Universidad de Antioquia, 43, 33-41.

Uby, L. (2019). Next steps in clean water oxygen transfer testing - A critical review of current standards. Water Research, 157, 415-434. Recuperado de https://doi.org/10.1016/j.watres.2019.03.063

United States Department of Agriculture \& Service, N. R. C. (July, 2011). Aeration of ponds used in aquaculture. Technical Note, Agricultural Engineering, (AEN 3), 1-15. 